\title{
Defects Generation and Surface Evolution of ZnO Nanobelts/Nanowires Under High-energy Electron Beam Irradiation
}

\author{
Yong Ding $^{1}$ and Zhong Lin Wang ${ }^{1}$ \\ 1. School of Materials Science and Engineering, Georgia Institute of Technology, Atlanta, GA, United \\ States
}

Using in situ transmission electron microscopy (TEM), we investigated the defect generation mechanism in $\mathrm{ZnO}$ nanobelts/nanowires under high-energy electron beam irradiation [1]. Further the dynamic reconstruction and evolution of $\mathrm{ZnO}$ polar and non-polar surfaces under high-energy electron beam irradiation were explored as well [2].

When $\mathrm{ZnO}$ nanobelts are exposed to a high-dose electron probe of several nanometers to hundred nanometers in diameter inside a transmission electron microscope, due to the radiolysis effect [3], part of oxygen atoms will be ejected into the vacuum and leaving a $\mathrm{Zn}$-ion rich surface with a pit appearance at both the electron-entrance and electron-exit surfaces. At the same time, a temperature distribution is created around the electron probe due to local beam heating effect, which generates a unidirectional pyroelectric field. This pyroelectric field is strong enough to drive $\mathrm{Zn}$ ions moving along its positive caxis direction as interstitial ions.

Convergent-beam electron diffraction (CBED) patterns in Fig. 1(b) are used to determine the positive caxis of the nanobelt in Fig. 1(a). The converged electron beam $(\sim 10 \mathrm{~nm}$ in diameter) induced defects due to the aggregation of $\mathrm{Zn}$ interstitial ions can be seen clearly in Fig. 1(c). Such defects were formed at some distances of 30-50 $\mathrm{nm}$ approximately along the c-axis direction away from the electron beam illuminated area. When we spread the electron beam to hundred nanometers scale, after several minutes, an arc shaped defect formed outside the electron-beam irradiated area as displayed in Fig. 1(d). Similarly, the direction from the center of the beam to the center of the defect arc is pointed along the positive c-axis. Such electron beam induced damage in $\mathrm{ZnO}$ nanostructures is suggested as a result of $\mathrm{Zn}$ ion diffusion driven by the temperature gradient induced pyroelectric field along its c-axis.

Electron beam radiolysis creates oxygen vacancies and a Zn rich (0001) surface as shown in the highresolution TEM (HRTEM) image in Fig. 2(a). The detailed distribution of the surface defects can be seen clearly in the atomic model in Fig. 2(c), which was used to simulate the HRTEM image in Fig. 2(b). Positive polar charges at the (0001) surface expel loosely bonded $\mathrm{Zn}$ ions to diffuse away from the (0001) polar surface. As a result, mass loss was observed around the (0001) surface. Dehydration by the electron beam breaks the charge balance on the $(000 \overline{1})$ polar surface. The negative charges on the $(000 \overline{1})$ surface suppress the radiolysis effect, and further absorb $\mathrm{Zn}$ ions to the surface to neutral the polar charges. Fig. 2(f) is a HRTEM image to show an individual $\mathrm{Zn}$ ion absorbed on the oxygen-terminated $(000 \overline{1})$ surface. The ideal stacking sequences of $\mathrm{Zn}$ ions in hexagonal $\mathrm{ZnO}$ structure can be considered as $\mathrm{ABAB} \ldots$ along its $\mathrm{c}$ axis, while the absorbed individual $\mathrm{Zn}$ ion on the $(000 \overline{1})$ surface occupies the $\mathrm{C}$ site as depicted in Fig. 2(d) and (e) to form three bonds with surface $\mathrm{O}$ ions beneath, instead of one bond in the ideal structure. The simulated image in Fig. 2(g) using the atomic model in Fig. 2 (d) has a good match with the experimental one in Fig. 2(f). With more Zn ion absorption and surface oxidization, new nanocrystals grow up from the $(000 \overline{1})$ polar surface. New nanocrystals nucleated at the $(01 \overline{1} 0)$ non-polar surface are driven by the electric field of the polar charges as well, for the $\mathrm{Zn}$ ions were always observed 


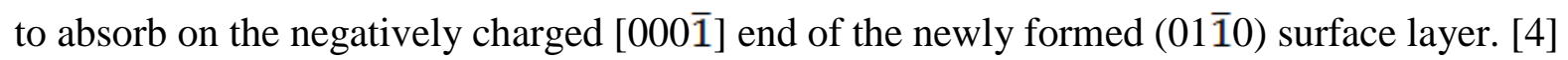

\section{References:}

[1] Y. Ding et al, J. Appl. Phys., 116 (2014), p.154304.

[2] Y. Ding, K. C. Pradel, and Z. L. Wang, J. Appl. Phys., 119 (2016), p. 015305.

[3] R. F. Egerton, P. Li, and M. Malac, Micron, 35 (2004), p. 399.

[4] The authors acknowledge funding support from National Science Foundation (DMR-1505319) and U.S. Department of Energy, Office of Basic Energy Sciences (Award DE-FG02-07ER46394).
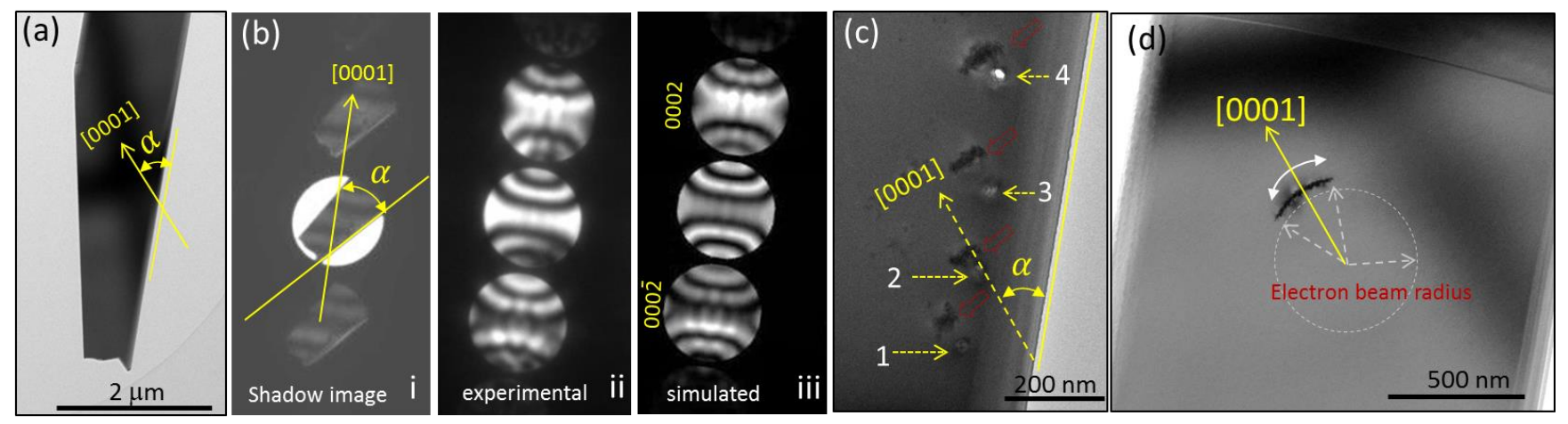

Figure 1. (a) A low-magnification bright-field TEM image to show a $\mathrm{ZnO}$ nanobelt with no electron beam damage yet. (b-i) A shadow image from the nanobelt in (a). (b-ii) and (b-iii) are experimental and simulated CBED pattern. (c) a TEM image shows the electron irradiation induced pits, holes and defects. (d) A bright-field TEM image to show the arc shaped defect formed due to electron beam irradiation with beam radius around $250 \mathrm{~nm}$.
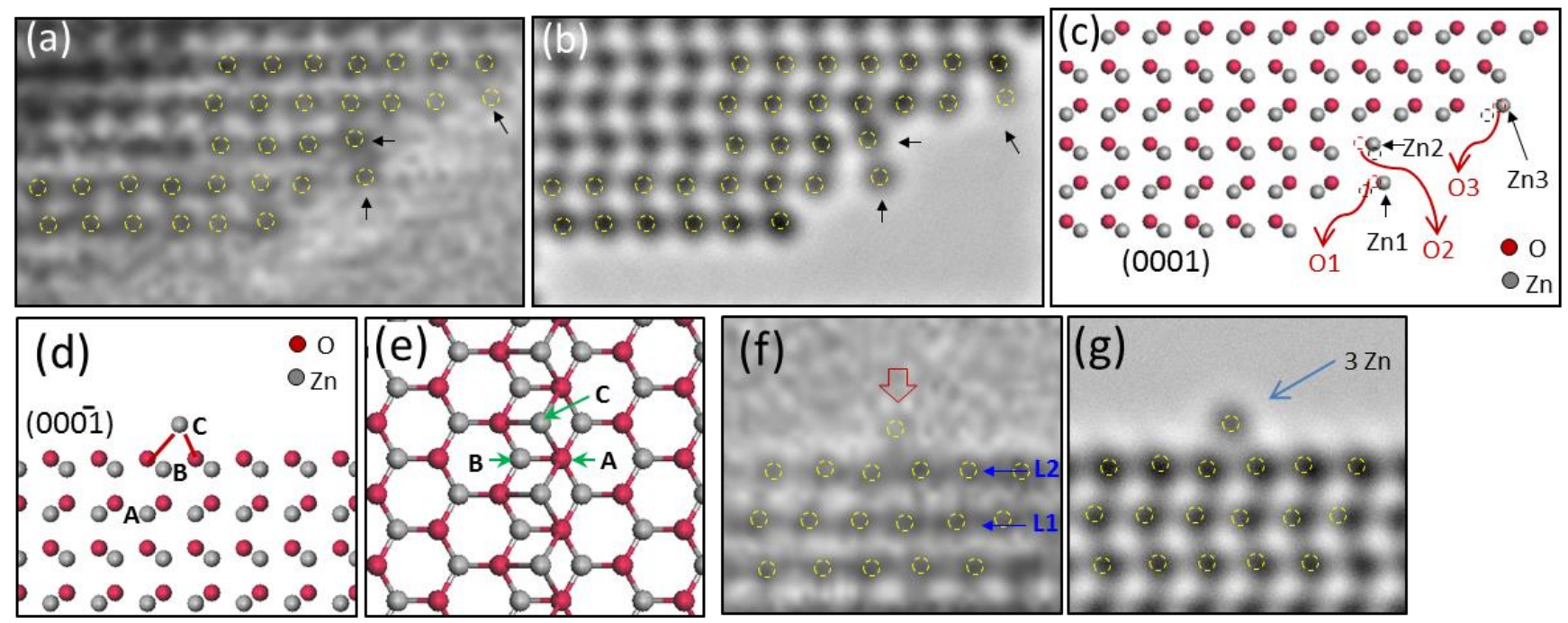

Figure 2. (a) A HRTEM image from the $\mathrm{ZnO}$ (0001) polar surface. (b) Simulated HRTEM image using the atomic model in (c). (d) and (e) Depict the atomic model projected from different orientation to show

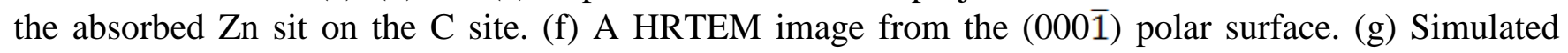
HRTEM image using the model in (d). 